\title{
Mixed-modality psychophysical scaling: Inter- and intramodality sequential dependencies as a function of lag
}

\author{
LAWRENCE M. WARD \\ University of British Columbia, Vancouver, British Columbia, Canada
}

\begin{abstract}
In mixed-modality psychophysical scaling, stimuli from different modalities are presented alternately for judgment on the same scale. This paper reports the results of two experiments using a variant of the standard technique that allowed investigation of inter- and intramodality sequential dependencies as a function of lag. Derived cross-modality matching functions from magnitude- and category-matching experiments were highly similar, with exponents around 1.6, as is the case for the standard technique. In both experiments, significant inter- and intramodality assimilation of responses to previous responses was found for lags of up to four trials back in the sequence of stimuli, whereas contrast of current responses to previous stimulus values occurred only within a modality, but also for lags of up to four trials. Second-order dependency of the correlation between current and previous responses as a function of the separation of their stimuli has the usual form (inverted v) for lags of up to four trials, both within and across modalities. Dependency of the coefficient of variation of the ratio of current to previous responses on the separation of their stimuli was found only within a stimulus modality, and only for a lag of one trial. These results strengthen the case for different sources for assimilative and contrastive sequential dependencies, and for the two second-order dependencies.
\end{abstract}

Mixed-modality psychophysical scaling involves making judgments of stimuli from two or more different sensory continua on the same scale. Because the judgments are made on the same scale, cross-modality matching functions can be generated indirectly by finding stimulus levels on different continua that give rise to judgments of the same magnitude. Stevens and Marks (1980) showed that the scaling task of magnitude estimation could be used for this purpose; they called the method "magnitude matching." Ward (1982) showed that category matching (i.e., using the method of category judgment in the mixedmodality context) and magnitude matching produce highly similar matching functions. Mixed-modality scaling may often be preferable to ordinary cross-modality matching because it employs responses that are easier to realize experimentally and that may be more natural (e.g., category judgments).

In addition to their uses as scaling instruments, mixedmodality methods produce data that are relevant to discriminating between theories of psychophysical judgment. Ward (1982), for example, reported that for both magnitude and category matching, current responses are assimilated to previous responses regardless of modality, whereas current responses are contrasted with intensities

This research was supported by Grant A9958 from the Natural Sciences and Engineering Research Council of Canada. The author thanks Barry Chew, Elsie Cheung, and Odie Geiger for assistance in collecting and analyzing the data. Send reprint requests to Lawrence $M$. Ward, Department of Psychology, University of British Columbia, 2136 West Mall, University Campus, Vancouver, BC V6T 1Y7, Canada. of previous stimuli of the same modality only. These data are consistent with theories in which the two types of sequential dependencies arise from different sources, and are inconsistent with theories in which both arise from the same source. The present paper reports data that extend the usefulness of mixed-modality scaling as an instrument and that answer questions raised by Ward (1982) regarding the effect of lag on inter- and intramodality sequential dependencies.

The standard mixed-modality method presents stimuli from different modalities for judgment in strict alternation. For example, if intensities of lights and sounds are being judged, a segment of a typical stimulus/response sequence would look like

$$
\ldots S_{n-3} R_{n-3} L_{n-2} R_{n-2} S_{n-1} R_{n-1} L_{n} R_{n} \ldots
$$

where $S_{n-k}$ represents a sound stimulus, $R_{n-k}$ a response, and $\mathrm{L}_{n-k}$ a light stimulus, on Trial $n-k$. In such sequences, intramodality effects must persist for at least two trials (e.g., a lag of 2) in order to be detectable on current responses, given that stimuli of the same modality are always separated by a stimulus (and its response) of a different modality. Because of this, Ward (1982) was unable to describe fully the effects of lag on the sequential dependencies he found. Furthermore, a wellconfirmed second-order dependency did not appear in Ward's data. For magnitude estimation data, the coefficient of variation (standard deviation/mean, or $\mathrm{s} / \mathrm{m}$ ) of the ratios of successive responses is smaller when the difference between the successive stimulus intensities, 
$I_{n}-I_{n-1}$, is small than when that difference is large. Ward (1982) reported that such an effect was found in his data neither within nor across modalities. However, as described above, intramodality effects with a lag of 1 could not be observed using the standard alternatingmodality stimulus/response sequence. It is possible that the typical $v$-shaped relation between $s / m$ and $I_{n}-I_{n-1}$ would be found within modalities for a lag of 1 . If so, the case (first made by Baird, Green, \& Luce, 1980) for the independence of this second-order dependency from the dependency of the correlation of successive responses on $I_{n}-I_{n-1}$ would be strengthened, disconfirming theories in which they arise from the same mechanism.

It is relatively simple to produce a stimulus/response sequence that allows both intra- and intermodality sequential dependencies to be studied for both short and long lags. An eight-trial segment of the sequence used in the present study is shown below.

$$
\begin{aligned}
& \ldots S_{n-7} R_{n-7} S_{n-6} R_{n-6} L_{n-5} R_{n-5} L_{n-4} R_{n-4} \\
& \quad S_{n-3} R_{n-3} S_{n-2} R_{n-2} L_{n-1} R_{n-1} L_{n} R_{n} \ldots,
\end{aligned}
$$

where $S_{n-k}, L_{n-k}$, and $R_{n-k}$ have the same meanings as in the typical sequence, presented above. In the sequence at hand, subjects make two successive judgments on one modality followed by two successive judgments on the other, and so forth. Here, intramodality effects for lags of 1 (e.g., $R_{n-7}$ on $R_{n-6}$ ), 3 (e.g., $R_{n-6}$ on $R_{n-3}$ ), 4 (e.g., $R_{n-6}$ on $R_{n-2}$ ), and so on can be detected, if present, as well as intermodality effects for lags of 1 (e.g., $R_{n-6}$ on $\mathbf{R}_{\mathbf{n}-5}$ ), 2 (e.g., $\mathbf{R}_{\mathbf{n}-6}$ on $\mathbf{R}_{\mathbf{n}-4}$ ), 3 (e.g., $\mathbf{R}_{\mathbf{n}-7}$ on $\mathbf{R}_{\mathbf{n}-4}$ ), and so on. This alternating-by-pairs sequence thus allows a relatively complete study of the effects of lag on both inter- and intramodality sequential dependencies. In addition to resolving the questions raised by Ward (1982), such a study could also contribute to the solution to the problem of whether effects for lags of more than one trial are important for theories of how psychophysical scaling judgments are made. Some authors have argued that effects for lags of more than one trial are unimportant (e.g., Jesteadt, Luce, \& Green, 1977), whereas others have found much more enduring effects (e.g., Staddon, King, \& Lockhead, 1980; Ward, 1982). Understanding the precise nature of the role of memory in scaling judgments depends in part on understanding the effects on the current response of stimuli and responses at different lags (cf. Lockhead \& King, 1983; Treisman \& Williams, 1984; Ward \& Lockhead, 1971).

Although this sequence may be useful for studying the effect of lag on sequential dependencies, it is still necessary to demonstrate that it produces scales comparable to those produced by the standard sequence. If it does, the usefulness of the mixed-modality scaling method will be increased as well, since other nonstandard sequences may be tried with greater confidence. This paper reports the results of experiments in magnitude and category matching using the alternating-by-pairs stimulus/response sequence described above. These results constitute the desired study of first- and second-order sequential dependencies as a function of lag, and they also establish the comparability of this sequence to the standard sequence.

\section{METHODS}

\section{Experiment 1: Magnitude Matching}

Subjects. Eight subjects, 6 females and 2 males, with normal or corrected-to-normal vision and no known hearing defects participated for pay.

Apparatus. An alternating-by-pairs sequence of sounds and lights was presented for judgment. The apparatus was identical to that of Ward (1982). Briefly, for the light stimuli, a $1.5^{\circ}$ dot of approximately uniform luminance was produced at about the eye level of the subject, who sat in a Model 250 IAC chamber. The 10 light intensities used were $167.6,84.00,42.10,21.10,10.58,5.300$, $2.656,1.331,0.667$, and $0.334 \mathrm{~cd} / \mathrm{m}^{2}$. Adjacent intensities were thus separated by $.3 \log$ units $(3 \mathrm{~dB})$. For the sound stimuli, a 1000 $\mathrm{Hz}$ tone was delivered diotically through stereo headphones at a sound pressure amplitude of $11.25,6.324,3.556,2.000,1.125$, $0.632,0.356,0.200,0.113$, or $0.063 \mathrm{dynes} / \mathrm{cm}^{2}$. Adjacent amplitudes were thus separated by $.25 \log$ unit $(5 \mathrm{~dB})$. These ranges of light and sound intensity are roughly equivalent in terms of proportions of the dynamic range, and are in the range of those used by Stevens and Marks (1980) in the first study of magnitude matching. Each stimulus was presented for $1 \mathrm{sec}$. A PDP 11/34A computer system controlled stimulus presentation and recorded responses, which were made on a standard computer keyboard illuminated by a dim red light.

Procedure. The procedure was identical to that used by Ward (1982), except for the stimulus/response sequence. Briefly, subjects made a total of 1,200 judgments (600 each of light and sound, about 60 per intensity level) in four sessions of 300 judgments each, after 200 practice judgments with light and sound and a few practice magnitude estimations of line lengths. Stimulus intensity was chosen at random on each trial. Subjects were dark-adapted for about $2 \mathrm{~min}$ before the start of each session. The intervals between light stimuli varied, from 5 to $7 \mathrm{sec}$ for successive judgments of lights to as long as 15-20 sec for judgments of lights separated by judgments of two sound stimuli. Thus the state of dark adaptation probably varied somewhat across judgments of light stimuli.

Subjects received instructions identical to those of Ward (1982), which were adapted from those of Stevens and Marks (1980). The instructions asked for magnitude estimations of lights and sounds on the same scale and emphasized that subjects were to attempt an "absolute" match of the number chosen to the sensation intensity of each stimulus. Subjects were told to expect the alternating-bypairs sequence.

\section{Experiment 2: Category Matching}

Subjects. Eight subjects, 5 males and 3 females, with normal or corrected-to-normal vision and no known hearing defects participated for pay. None of the subjects in Experiment 2 had participated in Experiment 1.

Apparatus. All stimuli and equipment were identical to those used in Experiment 1.

Procedure. The procedure was identical to that of Experiment 1 except that subjects made category judgments instead of magnitude estimations of the stimuli, and the practice session was changed to accommodate the different method, similarly to Experiment 2 of Ward (1982). Instructions were identical to those given by Ward, except that subjects were told to expect the alternating-by-pairs sequence instead of the standard sequence. Briefly, subjects were instructed to place the sensory intensities generated by the various stimuli into 1 of 10 categories, again emphasizing that the category scale used should be one common to the light and sound stimuli. 


\section{RESULTS AND DISCUSSION}

\section{Cross-Modality Matching Functions}

Figure la shows the cross-modality matching function for light and sound intensity generated from the data of Experiment 1 using the procedure of Stevens and Marks (1980). Individual cross-modality matching functions were highly similar to this average function. All matching functions were reasonably well fitted by simple power functions (straight lines in decibel coordinates). The slopes of the matching functions for individual subjects averaged 1.74 , which is close to the slope of 1.69 for the average matching function exhibited in Figure 1a, and to the corresponding slopes of 1.60 and 1.60 obtained by Ward (1982). (Note that the equations shown in Figure 1 represent the regression of sound-on-light only, whereas the overall slopes reported are the geometric means of the sound-on-light slopes and the reciprocal of the lighton-sound slopes as reported by Stevens and Marks, 1980, Appendix 2.) It is clear that the alternating-by-pairs sequence produced highly similar matching functions to those produced by the standard sequence.

Figure $1 \mathrm{~b}$ shows the average cross-modality matching function obtained from the category-matching data of Experiment 2 . Individual subjects' functions were highly similar to the group function. The slopes of the matching functions for the individual subjects averaged 1.57 , close to the slope of 1.60 obtained for the averaged data and to the corresponding values of 1.52 and 1.47 obtained by Ward (1982). Moreover, as found by Ward, the average slope of the individual category-matching functions is not reliably different from that of the individual magnitudematching functions $[t(14)=1.233]$. Thus, the data from these experiments using the alternating-by-pairs sequence replicate Ward's data very closely in regard to crossmodality matching functions. This means that mixedmodality scaling is not highly sensitive to the particular sequence of modalities, and that conclusions drawn about the effects of lag on sequential dependencies in these experiments can be generalized at least to other mixedmodality situations.

\section{Sequential Dependencies}

Multiple regression analyses. Several authors have argued that a useful summary of sequential dependencies in scaling data is provided by estimation of $\gamma, \alpha_{\mathrm{i}}$, and $\beta_{\mathrm{k}}$ in

$$
\begin{aligned}
\log R_{n}= & \gamma \log I_{n}+\sum_{i=1}^{M} \alpha_{i} \log I_{n-i} \\
& +\sum_{k=1}^{N} \beta_{k} \log R_{n-k}+\delta+\epsilon
\end{aligned}
$$

(see, e.g., Jesteadt et al., 1977; Ward, 1979). The constants in Equation 1 were estimated by hierarchical linear regression, in which the variables were entered in the order in which they occurred in the stimulus/response se- quence, for example in the order $I_{n}, R_{n-1}, I_{h}, R_{n-2}, I_{n-2}$, and so forth. For the analyses reported here, $\mathrm{M}$ and $\mathrm{N}$ were set equal to 4 , and the estimates of the coefficients are reported only for a lag of up to 4 , because individual subjects' coefficients were seldom reliable for higher lags. Because it is not clear what to make of the reliability of these estimates for individual subjects' data, conclusions are based mostly on $t$ tests of average estimates over the group of subjects and on the average $\mathbf{R}^{2}$ increase for each independent variable.

Tables 1 and 2 summarize the results of the multiple regression analyses on the data of Experiment 1. The averages of the estimates of the coefficients of Equation 1 for individual subjects' data are shown in Table 1, and the averages of the $\mathrm{R}^{2}$ increase for the corresponding independent variables are shown in Table 2 . In interpreting the entries in Tables 1 and 2 , remember that light and sound alternate by pairs in the sequence of trials as described in the introduction. Furthermore, responses to light and sound stimuli are analyzed separately, as are responses to the first and second members of each pair of stimuli of the same modality. Thus, the row labels in Tables 1 and 2 refer to the responses to the stimuli portrayed in the sequence

$$
\ldots S_{1} S_{2} L_{1} L_{2} S_{1} S_{2} L_{1} L_{2} \ldots
$$

Thus, for $R S_{1}, I_{n}$ is a sound stimulus, $R_{n-1}$ is a response to a light stimulus $\left(L_{2}\right), I_{n-1}$ is that light stimulus $\left(L_{2}\right)$, $R_{n-2}$ is a response to a light stimulus $\left(L_{1}\right), I_{n-2}$ is that light stimulus, and so forth.

Look carefully at Row $L_{1}$ in Table 1 . This row exemplifies the most common pattern of regression coefficients in terms of which ones are reliably different from zero. Clearly, $\gamma$ is large and reliably different from zero; it represents the standard power function exponent, but with other influences partialled out. Because $I_{n}$ for the $L_{1}$ sequence is a light stimulus, $\gamma$ represents the exponent for light, which is roughly the same as the standard value of .33 for that exponent. Now notice that $\beta_{1}$ and $\beta_{2}$ are reliably different from zero. The positive value of these coefficients means that responses on Trials $n-1$ and $n-2$ had an assimilative effect on the response on Trial n. $\beta_{1}$ and $\beta_{2}$ represent intermodality effects, because they represent the effect of responses to $S_{2}$ and $S_{1}$. Now consider the $\alpha$ coefficients in Row $\mathrm{L}_{1}$. Notice that only $\alpha_{3}$ and $\alpha_{4}$ are reliably different from zero. Both of these represent intramodality effects, from previous $L_{2}$ and $L_{1}$, respectively. Their negative signs indicate that responses to the current $L_{1}$ are contrasted with the values of the previous $L_{2}$ and $\mathrm{L}_{1}$.

Analyzing the other rows of regression coefficients in Table 1 in the same manner reveals that there is only one exception to the pattern of both inter- and intramodality effects of previous responses, but of only intramodality effects of previous stimuli. The exception is $\alpha_{2}$ in Row $S_{1}$. It is an example of a (marginally) reliable intermodality stimulus effect. However, note that the coefficient is about 


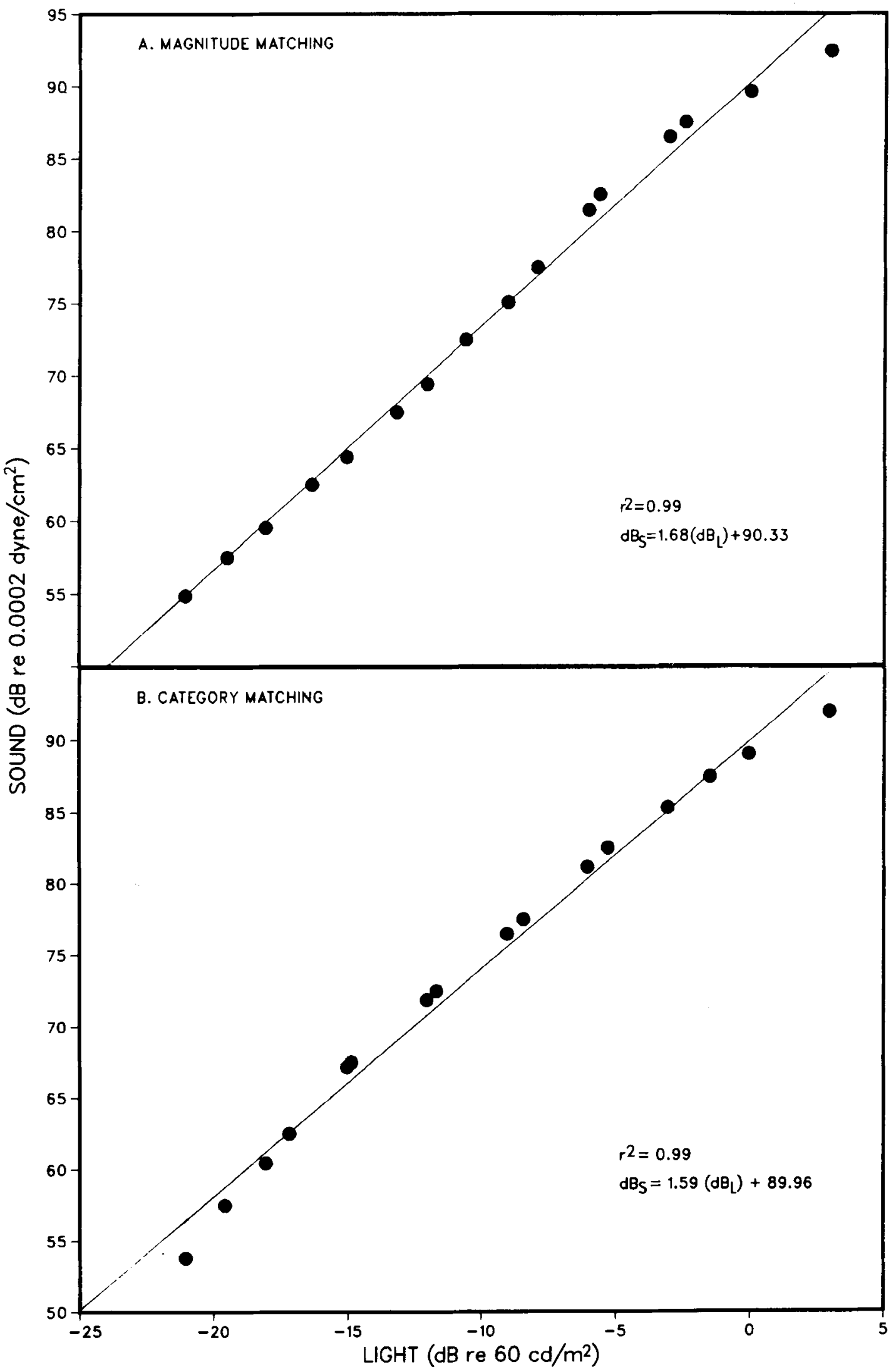

Figure 1. Cross-modality matching functions generated from the magnitude-matching and category-matching data. The straight lines through the points are the lines whose equations are printed on the figure. 
Table 1

Regression Coefficients from Equation 1 for Magnitude-Matching Data

\begin{tabular}{|c|c|c|c|c|c|c|c|c|c|}
\hline & $\gamma$ & $\beta_{1}$ & $\alpha_{1}$ & $\beta_{2}$ & $\alpha_{2}$ & $\beta_{3}$ & $\alpha_{3}$ & $\beta_{4}$ & $\alpha_{4}$ \\
\hline$\overline{S_{1}}$ & $.415^{*}$ & $.147^{*}$ & -.020 & $.080^{\dagger}$ & $-.021 \dagger$ & $.074 \dagger$ & $-.077^{*}$ &, $077 \dagger$ & $-.060 *$ \\
\hline $\mathrm{S}_{2}$ & $.387^{*}$ & $.313^{*}$ & -.072 & .042 & -.008 & .024 & -.009 & $.058^{*}$ & $-.038 *$ \\
\hline $\mathrm{L}_{\mathrm{k}}$. & $.327^{*}$ & $.117^{*}$ & -.023 & $.079 *$ & -.021 & .102 & $-.064 \dagger$ & .081 & $-.049 *$ \\
\hline $\mathrm{L}_{2}$ & $.321^{*}$ & $.309^{*}$ & $-.082 *$ & .017 & -.018 & .039 & -.031 & $.095 \dagger$ & $-.055^{*}$ \\
\hline
\end{tabular}

Table 2

$R^{2}$ Increase from Equation 1 for Magnitude-Matching Data

\begin{tabular}{lccccccccc}
\hline & $\mathbf{I}_{\mathbf{n}}$ & $\mathbf{R}_{\mathbf{n}-1}$ & $\mathbf{I}_{\mathbf{n}-1}$ & $\mathbf{R}_{\mathbf{n}-2}$ & $\mathbf{I}_{\mathbf{n}-2}$ & $\mathbf{R}_{\mathbf{n}-3}$ & $\mathbf{I}_{\mathbf{n}-3}$ & $\mathbf{R}_{\mathbf{n}-4}$ & $\mathrm{I}_{\mathbf{n}-4}$ \\
\hline $\mathrm{S}_{1}$ & .741 & .013 & .002 & .002 & .000 & .003 & .008 & .002 & .003 \\
$\mathrm{~S}_{2}$ & .692 & .043 & .008 & .002 & .001 & .001 & .001 & .001 & .002 \\
$\mathrm{~L}_{1}$ & .739 & .015 & .004 & .003 & .001 & .001 & .008 & .002 & .003 \\
$\mathrm{~L}_{2}$ & .708 & .028 & .015 & .002 & .000 & .004 & .001 & .001 & .004 \\
\hline
\end{tabular}

the same size as other coefficients that are not reliably different from zero. It is doubtful that it is a replicable effect.

Another interesting aspect of Table 1 is that the values of $\beta_{1}$ in Rows $S_{2}$ and $L_{2}$ are considerably larger than those in Rows $S_{1}$ and $L_{1}$. These differences are statistically reliable $[\mathrm{t}(14)=2.728$ and $2.837, \mathrm{p}<.02$, for sound and light, respectively]. The larger values of $\beta_{1}$ are for intramodality situations. Previous responses to stimuli of the same modality seem more influential than previous responses to stimuli of a different modality, although both are quite reliable.

We can obtain an impression about how far back in the stimulus/response sequence such effects must be considered important by juxtaposing the regression coefficients with the $\mathbf{R}^{2}$ increase for their respective independent variables, displayed in Table 2 . It is clear that in every case, $I_{n}$ accounts for the bulk of the variance in subjects' responses, as it should if these are to be useful scaling methods. Also, consistent with the conclusions of Jesteadt et al. (1977), $R_{n-1}$ and $I_{n-1}$ account for significant amounts of variance, although $R_{n-1}$ is not as important as $I_{n-1} \cdot R_{n-1}$ increases in importance when it is made to a stimulus of the same modality, and $I_{n-1}$ is more important when an intramodality stimulus contrast effect is present. The effects of responses at longer lags are not as important, seldom accounting for even $0.5 \%$ of the response variance on the average, but are still reliable and may be theoretically important. Most surprising are the intramodality stimulus contrast effects, which are quite reliable and result in increases in $\mathrm{R}^{2}$ of almost $1 \%$ at a lag of 3 , and are still highly reliable, although with a lower $R^{2}$ increase, at a lag of 4 . In this situation, a lag of 4 represents an interstimulus duration of roughly $20 \mathrm{sec}$. These are variance sources that must be addressed by theory. Furthermore, it is difficult to see how such enduring effects could be attributed to transient sensory memory systems. Theories such as those of Lockhead and King (1983), in which longer term memory systems are implicated, seem to be required to account for this effect.
In summary, these data replicate those of Ward (1982) in that the effects of previous responses on current responses were found to be assimilative and to occur both within and across modalities, whereas the effect of previous stimuli on current responses was contrastive, and this effect occurred reliably only within stimulus modalities. Both types of effects were reliable for lags of up to 4 (and seldom for higher lags), indicating that enduring memory traces for stimuli and responses must be involved in magnitude-estimation judgments of light and sound intensity within the mixed-modality situation.

Tables 3 and 4 display the results of a similar regression analysis for the category-matching data from Experiment 2 . Inspection of Tables 3 and 4 reveals that the conclusions stated above for magnitude matching hold for category matching as well. Only a single exception was found to the pattern of reliable inter- and intramodality assimilative response effects and reliable intramodality contrastive stimulus effects. In Table 3 , in Row $S_{1}, \alpha_{1}$ is reliably different from zero for these data. Again, it is about the same size as other coefficients that are not reliably different from zero, as for the exception noted in the magnitude-matching data, indicating that it is of dubious reliability. Again, important effects were found for lags of up to 4 , indicating that the sources of these effects must be taken seriously in attempts to describe the processes involved in making such psychophysical judgments. Finally, the differences, represented in Table 3, between $\beta_{1}$ for Rows $S_{2}$ and $L_{2}$ and Rows $S_{1}$ and $L_{1}$ were statistically reliable $[\mathrm{t}(14)=3.382, \mathrm{p}<.01$, and 6.241 , $\mathrm{p}<.001$, for sound and light, respectively].

One other aspect of the regression coefficients that deserves mention is the relationship of the values of the coefficients to the standard power function exponents and across the current experiments. As mentioned above, the exponent for light from magnitude matching is only slightly lower than that usually found in magnitudeestimation experiments. However, that for sound is considerably lower than the usual value of around 0.67 for sound pressure. This may have been caused by the re- 
Table 3

Regression Coefficients from Equation 1 for Category-Matching Data

\begin{tabular}{llllllllll}
\hline & $\gamma$ & $\beta_{1}$ & $\alpha_{1}$ & $\beta_{2}$ & $\alpha_{2}$ & $\beta_{3}$ & $\alpha_{3}$ & $\beta_{4}$ & $\alpha_{4}$ \\
\hline $\mathrm{S}_{1}$ & $.381^{*}$ & $.121^{*}$ & $-.016 \dagger$ & .050 & -.008 & $.120^{*}$ & $-.089^{*}$ & $.076^{*}$ & $-.053^{*}$ \\
$\mathrm{~S}_{2}$ & $.371^{*}$ & $.366^{*}$ & $-.137 \dagger$ & $.040 \dagger$ & -.003 & .060 & -.021 & $.080^{*}$ & $-.056^{*}$ \\
$\mathrm{~L}_{1}$ & $.294^{*}$ & $.117^{*}$ & -.021 & .040 & -.006 & .065 & $-.044^{*}$ & $.095^{*}$ & $-.050^{*}$ \\
$\mathrm{~L}_{2}$ & $.294^{*}$ & $.272^{*}$ & $-.060^{*}$ & .038 & -.015 & .003 & -.006 & $.053 \dagger$ & $-.036^{*}$ \\
\hline${ }^{*} p<.01$ by t test. $\dagger p<<.05$ by $t$ test. & & & & & &
\end{tabular}

Table 4

$R^{2}$ Increase from Equation 1 for Category-Matching Data

\begin{tabular}{lccccccccc}
\hline & $\mathrm{I}_{\mathbf{n}}$ & $\mathrm{R}_{\mathbf{n}-1}$ & $\mathrm{I}_{\mathbf{n}-1}$ & $\mathbf{R}_{\mathbf{n}-2}$ & $\mathbf{I}_{\mathbf{n}-2}$ & $\mathbf{R}_{\mathbf{n}-3}$ & $\mathbf{I}_{\mathbf{n}-3}$ & $\mathbf{R}_{\mathbf{n}-4}$ & $\mathbf{I}_{\mathbf{n}-4}$ \\
\hline $\mathrm{S}_{1}$ & .802 & .007 & .002 & .002 & .001 & .004 & .012 & .002 & .003 \\
$\mathrm{~S}_{2}$ & .772 & .012 & .030 & .002 & .000 & .000 & .002 & .002 & .004 \\
$\mathrm{~L}_{1}$ & .777 & .008 & .002 & .002 & .001 & .003 & .006 & .002 & .006 \\
$\mathrm{~L}_{\mathbf{2}}$ & .768 & .021 & .011 & .000 & .001 & .000 & .000 & .002 & .004 \\
\hline
\end{tabular}

quirement of judging the two continua on the same scale. Also, although somewhat lower than the magnitudematching exponents, those for category-matching do not display the 1:2 ratio that category-judgment exponents typically have to magnitude-estimation exponents for the same continua (Marks, 1974; Ward, 1971, 1974). The interpretation of this finding will depend on how the typical result is explained, and there is no single, universally accepted explanation. Purely empirically, similar exponents arise, given equal stimulus ranges, when similar response ranges have been employed (see, e.g., King \& Lockhead, 1981). In the present case, it would seem that the magnitude-estimation responses were more restricted in range than is usual. The question remaining is why this happened, and the answer awaits further research.

Second-order dependencies. In magnitude-estimation data the relation between $R_{n}$ and $R_{n-k}$ has often been found to depend on the separation between $I_{n}$ and $I_{n-k}$ (or between $S_{n}$ and $S_{n-k}$ ). Jesteadt et al. (1977) found that the correlation between $\log R_{n}$ and $\log R_{n-1}$ was largest when $I_{n}-I_{n-1}$ was near zero and smallest when $I_{n}-$ $I_{n-1}$ was different from zero. Ward (1982) found the same pattern for both category-matching and magnitudematching data, both within and between modalities. Figures 2 and 3 display the results of analyses of the present data for these second-order dependencies. Only results for lags of 1 and 3 are displayed; lags of 2 and 4 give highly similar results and are not displayed. Note that $S_{n}-S_{n-1}$ is given in nominal steps; the difference between adjacent stimulus intensities was equal in log units. Notice also that in both figures the analysis extends only to absolute values of $S_{n}-S_{n-1}$ less than or equal to 5 . This is because these are the averages of individual subjects' correlations, and the individual subjects' correlations for greater step sizes were seldom based on enough observations to be reliable, or in some cases even calculable. Each of the averages in Figures 2 and 3 is based on at least five individual subjects' correlations, and each correlation was calculated on at least 15 pairs of values.

The message of Figures 2 and 3 is clear. For lags of up to 4 , and for both category- and magnitude-matching data, the correlation between $R_{n}$ and $R_{n-k}$ depends on $S_{n}-S_{n-k}$ in the same way as has always been found before. Clearly, both first- and second-order assimilative response-response sequential dependencies are independent of which modality is being responded to.

Variability of response ratios. As mentioned in the introduction, the coefficient of variation for ratios of successive magnitude estimations, $\mathrm{s} / \mathrm{m}$, has been found to vary as a function of $I_{n}-I_{n-1}$ (e.g., Green \& Luce, 1974). Ward (1982) did not find this result either for magnitude or category matching. However, that study did not investigate intramodality effects for a lag of 1 . Figures 4 and 5 display the relationship between $\mathrm{s} / \mathrm{m}$, calculated for $R_{n} / R_{n-k}$, and $S_{n}-S_{n-k}$ in nominal steps, for the magnitude- and category-matching data, respectively. As in Figures 2 and 3, the values plotted are averages of individual subjects' values, with each point representing at least 5 subjects' data. The upper and lower parts of Figures 4 and 5 represent lags of 1 and 3, respectively; the results for lags of 2 and 4 closely resemble those for the lag of 3 and are thus not displayed.

Consider Figure 4. Although the variability of the individual values of $\mathrm{s} / \mathrm{m}$ is large, it is clear that the expected $v$-shaped pattern does not emerge for either intra- or intermodality comparisons for a lag of 3 , and thus also not for lags of 2 or 4 . This is consistent with the results of Ward (1982). In the upper part of Figure 4, however, there is a suggestion of a $v$-shaped plot for intramodality $s / m\left(L_{1}\right.$ on $L_{2}$ and $S_{1}$ on $\left.S_{2}\right)$, but not for intermodality $\mathrm{s} / \mathrm{m}\left(S_{2}\right.$ on $L_{1}$ and $L_{2}$ on $\left.S_{1}\right)$. To see this more clearly, draw a straight line between the points at the ends of the plot and note that most intervening points lie below that line for the intramodality plots, but not for the intermodality plots. Because the variability of individual subjects' $\mathrm{s} / \mathrm{m}$ values is so large, this is not really clear-cut, but at least it indicates that, if there is such an effect in these data, it occurs only within stimulus modalities and for a lag of 1 . This strengthens the claim that the effect is unrelated to the assimilative response-response dependencies, which occur both within and between modalities. The effect could be the result of a mechanism that makes 

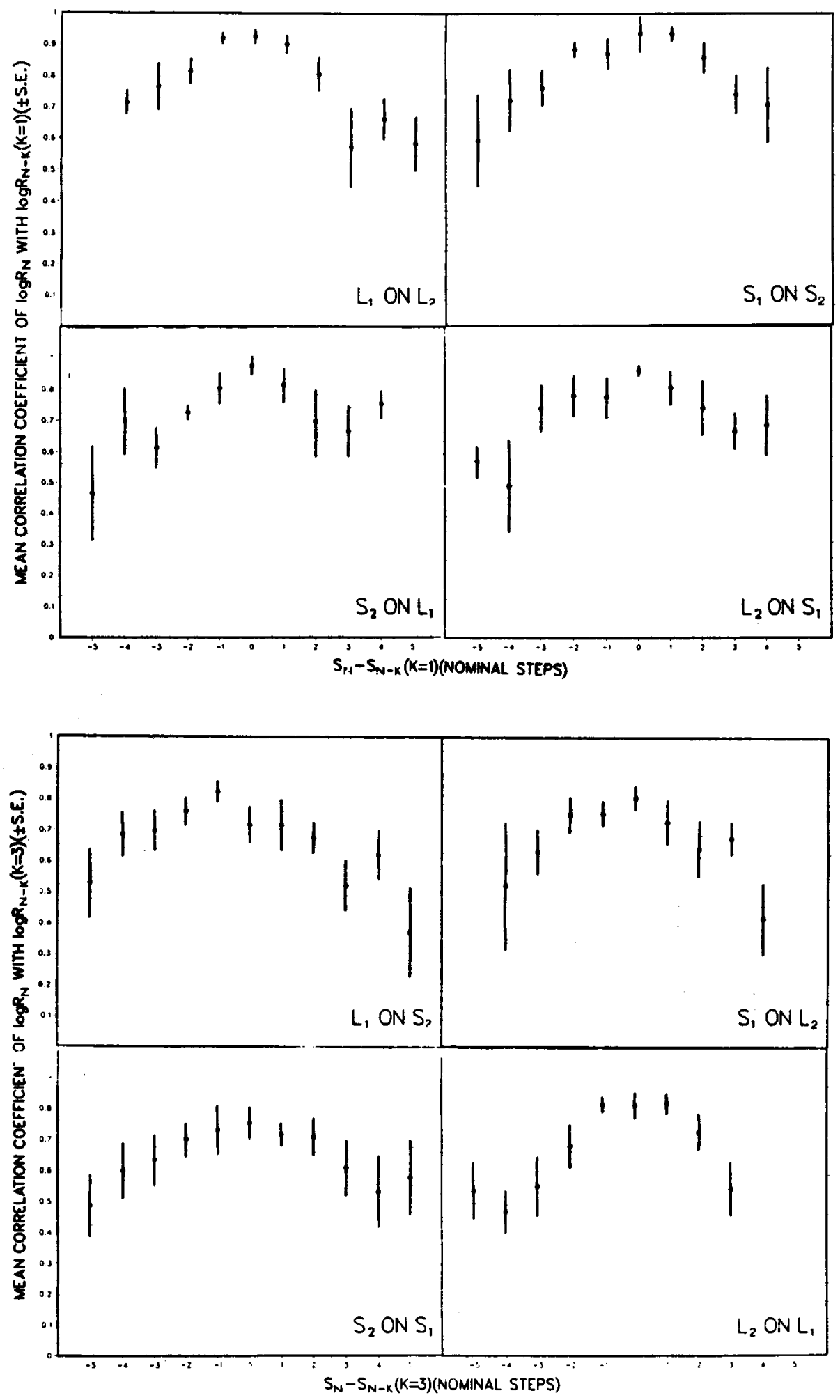

Figure 2. Plots of the correlation between $\log R_{n}$ and $\log R_{n-k}$ as a function of $S_{n}-S_{n-k}$ for lags of $1(k=1)$ and $3(k=3)$ for the magnitude-matching data. 

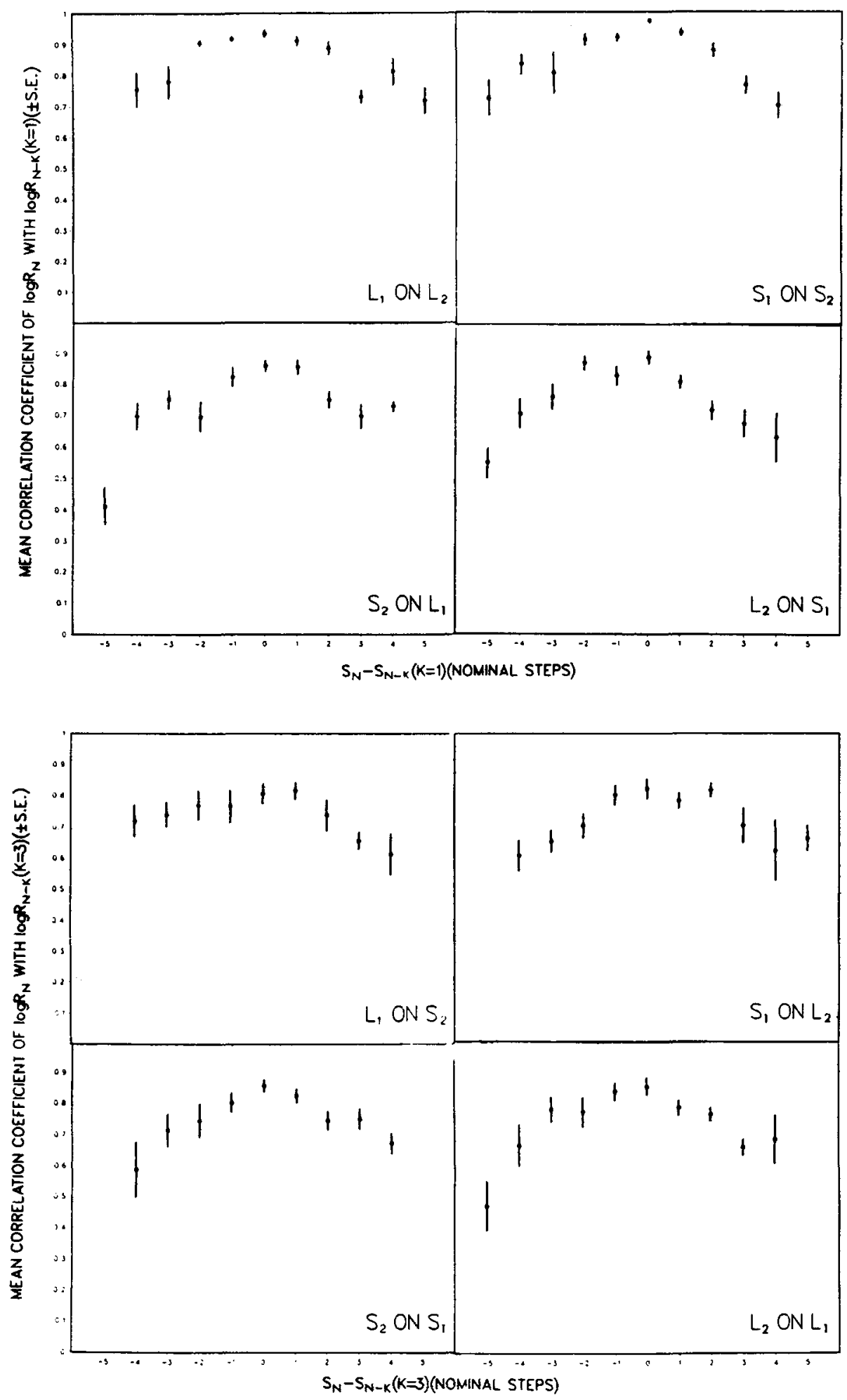

Figure 3. Plots of the correlation between $\log R_{n}$ and $\log R_{n-k}$ as a function of $S_{n}-S_{n-k}$ for lags of $1(k=1)$ and $3(k=3)$ for the category-matching data. 

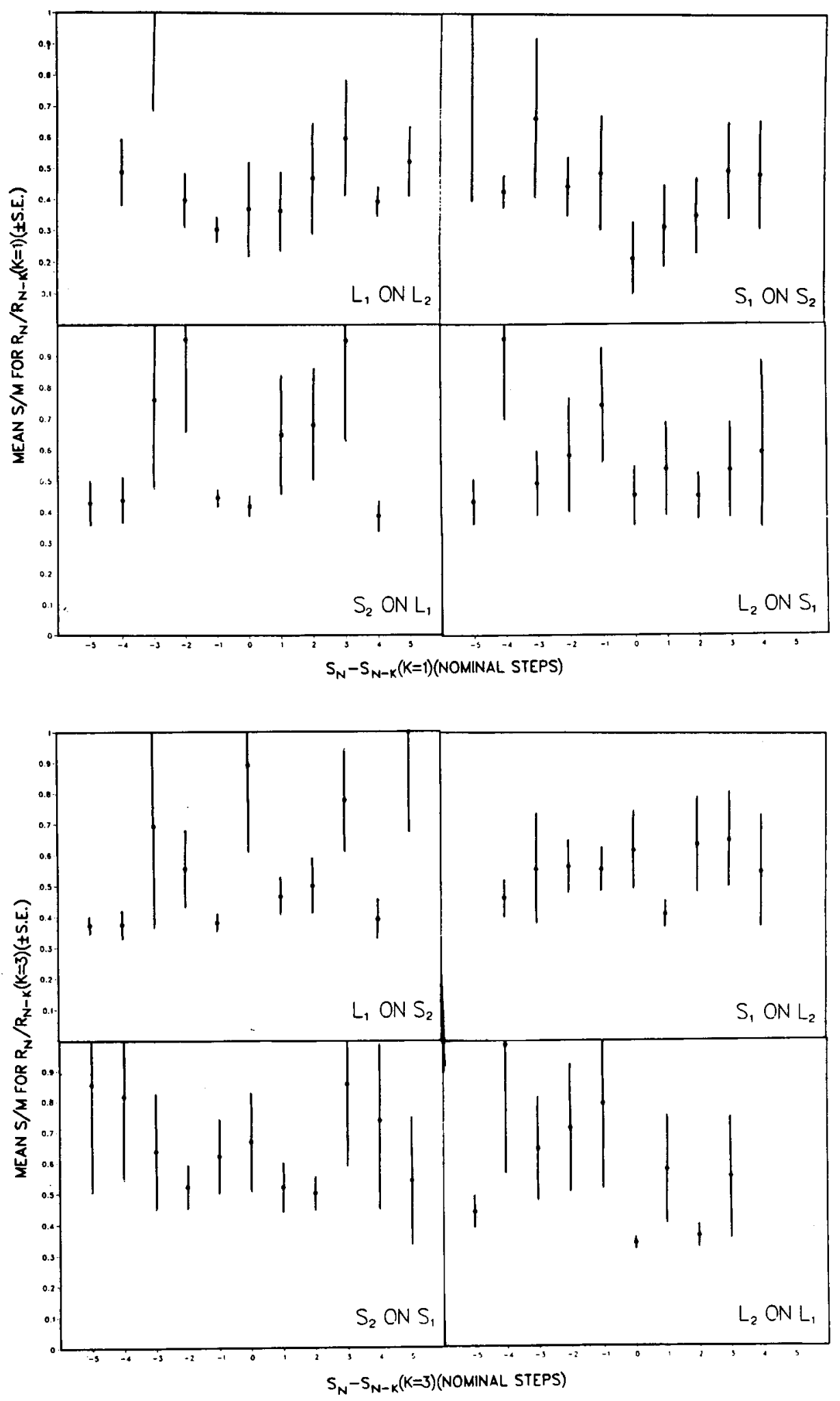

Figure 4. Plots of $s / m$ for $R_{n} / R_{n-k}$ as a function of $S_{n}-S_{n-k}$ for lags of $1(k=1)$ and $3(k=3)$ for the magnitude-matching data. 

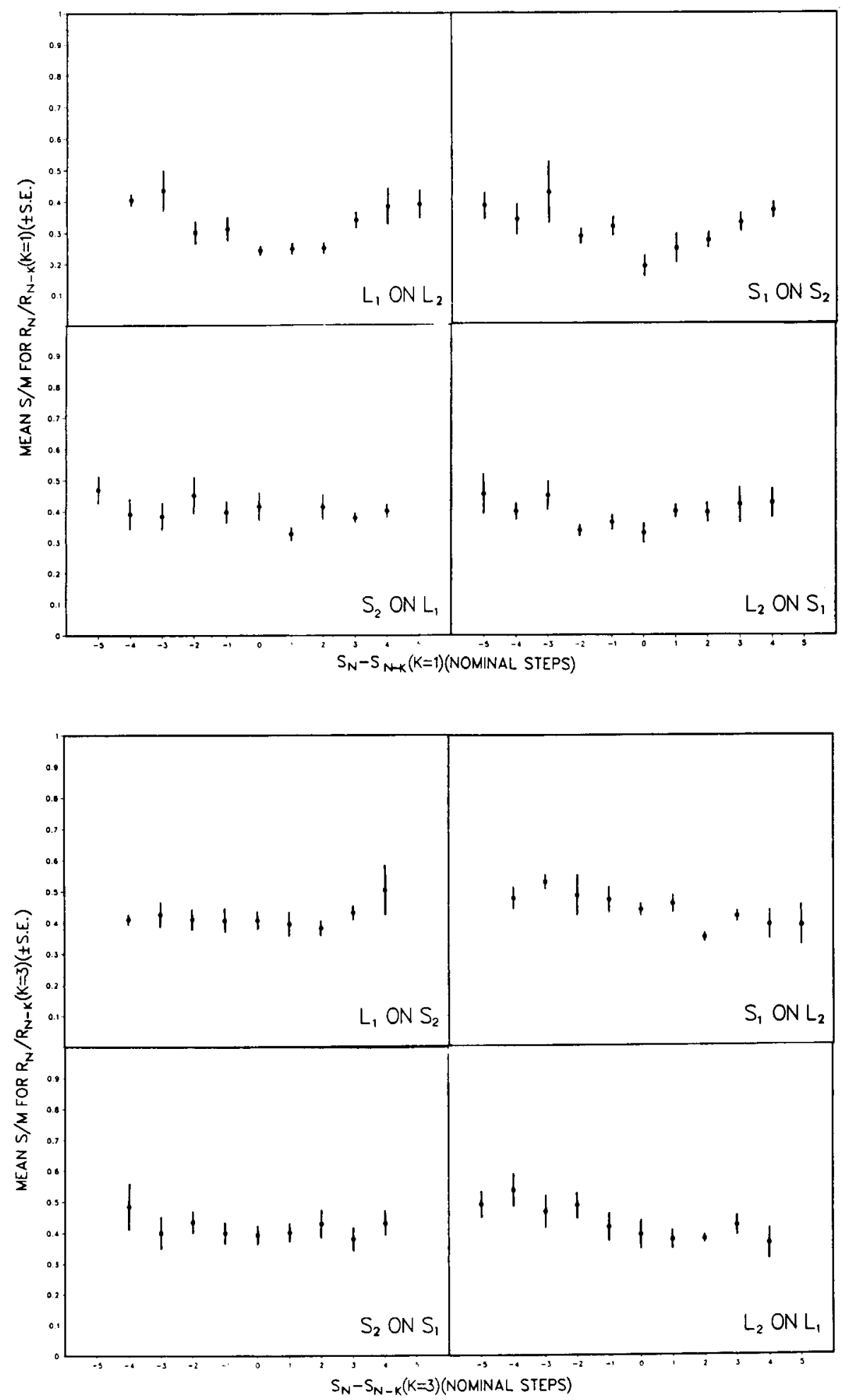

Figure 5. Plots of $s / m$ for $R_{n} / R_{n-k}$ as a function of $S_{n}-S_{n-k}$ for lags of $1(k=1)$ and $3(k=3)$ for the category-matching data. 
the internal representation of the stimulus more precise, or more stable, when it is near the previous stimulus value on the same modality. An example of such a mechanism is the attention band, suggested by Green and Luce (1974), within which a sample of neural interarrival times representing the stimulus intensity is larger than without. Although other mechanisms could be proposed to accomplish the same end, it is clear that all of them must have the property of being modality-specific and interfered with by intervening stimuli and/or judgments.

Now consider Figure 5. The data from the categorymatching task strongly resemble those from magnitude matching, except that the variability of individual subjects' $\mathrm{s} / \mathrm{m}$ values is much lower, and so the pattern is clearer. For a lag of 1 and within modality, there is a v-shaped relation between $\mathrm{s} / \mathrm{m}$ and $S_{n}-S_{n-k}$. For all other plots, with the possible exception of $L_{2}$ on $S_{1}$ for $k=1$, there is no suggestion of such an effect. This is also true for lags of 2 and 4. Again, the dependency of $s / m$ on $S_{n}-$ $S_{n-k}$ seems to occur mostly within modalities and for a lag of 1 . The intermodality effect for $k=1$ is both quite weak and unique, in that Ward (1982) found no such intermodality effect for a lag of 1 in data in which each $\mathrm{s} / \mathrm{m}$ was based on a higher number of judgments. It is therefore possibly not reliable, and its importance remains to be established.

\section{CONCLUSIONS}

The data reported in this paper strengthen the notion that there are several mechanisms responsible for sequential dependencies in magnitude-estimation and categoryjudgment data. Assimilative response-response dependencies of both the first and second orders arise both within and between stimulus modalities in mixed-modality tasks, whereas contrastive response-stimulus dependencies arise only within modalities in such tasks. Such dependencies are reliable up to lags of at least 4 , and the effects for lags greater than 1 are probably important enough to be addressed in theory. This is especially true for contrastive effects, which are surprisingly strong even at lags of 4 and demand an explanation for such persistence. Moreover, the dependency of $s / m$ for ratios of responses at various lags on $S_{n}-S_{n-k}$ seems to occur only within modalities for lags of 1 . This effect is thus separable from both first- and second-order assimilative responseresponse dependencies and from contrastive response- stimulus dependencies, which occur in lags up to at least 4 as well. The picture that emerges from these data is of at least three different mechanisms giving rise to the three types of effect. Furthermore, as pointed out by a reviewer, both temporal (e.g., lag) and spatial (e.g., intensity) proximity play roles in these dependencies. More empirical and theoretical study is necessary to converge on convincing accounts of how such mechanisms work together to produce judgments of sensory intensity that display these complex properties.

\section{REFERENCES}

BaIRD, J. C., Green, D. M., \& LUCE, R. D. (1980). Variability and sequential effects in cross-modality matching of area and loudness. Journal of Experimental Psychology: Human Perception \& Performance, 6, 277-289.

Green, D. M., \& LuCE, R. D. (1974). Variability of magnitude estimates: A timing theory analysis. Perception \& Psychophysics, 15, 291-300.

Jesteadt, W., Luce, R. D., \& Green, D. M. (1977). Sequential effects in judgments of loudness. Journal of Experimental Psychology: Human Perception \& Performance, 3, 92-104.

KING, M. C., \& LockHEAD, G. R. (1981). On memory effects in magnitude estimation experiments. Perception \& Psychophysics, 30 , 599-603.

LockheAD, G. R., \& KiNG, M. C. (1983). A memory model of sequential effects in scaling tasks. Journal of Experimental Psychology: Human Perception \& Performance, 9, 461-473.

MarKs, L. E. (1974). On scales of sensation: Prolegomena to any future psychophysics that will be able to come forth as science. Perception \& Psychophysics, 16, 358-376.

Staddon, J. E. R., King, M. C., \& Lockhead, G. R. (1980). On sequential effects in absolute judgment experiments. Journal of Experimental Psychology: Human Perception \& Performance, 6, 290-301.

Stevens, J. C., \& MARKs, L. E. (1980). Cross-modality matching functions generated by magnitude estimation. Perception \& Psychophysics, 27, 379-389.

Treisman, M., \& Williams, T. C. (1984). A theory of criterion setting with an application to sequential dependencies. Psychological Review, 91, 68-111.

WARD, L. M. (1971). Some psychophysical properties of category judgments and magnitude estimations. Dissertation Abstracts International, 32, 1887B. (University Microfilms No. 71-24, 212)

WARD, L. M. (1974). Power functions for category judgments of duration and line length. Perceptual \& Motor Skills, 38, 1182.

WARD, L. M. (1979). Stimulus information and sequential dependencies in magnitude estimation and cross-modality matching. Journal of Experimental Psychology: Human Perception \& Performance, 5 , 444-459.

WARD, L. M. (1982). Mixed-modality psychophysical scaling: Sequential dependencies and other properties. Perception \& Psychophysics, 31 , 53-62.

WARD, L. M., \& LOCKHEAD, G. R. (1971). Response system processes in absolute judgment. Perception \& Psychophysics, 9, 73-78. 